\title{
Prediction of the onset of atrial fibrillation after cardiac surgery using the monophasic action potential
}

\author{
A M Pichlmaier, V Lang, W Harringer, B Heublein, M Schaldach, A Haverich
}

\begin{abstract}
Objective-To show that the monophasic action potential (MAP) recorded continuously from human epicardium may be used to predict the imminent onset of atrial fibrillation or flutter (AF) following surgery, thus allowing prophylactic treatment to be started.
\end{abstract}

Patients-22 patients (14 male, 8 female; mean (SD) age 64 (12) years) undergoing aortic valve replacement.

Setting-Tertiary referral centre.

Methods-Over a mean observation period of 8 (2.7) days (range 4 to 14), nine episodes of AF were seen in six patients. Before AF, specific and significant alterations of the MAP morphology were observed. In seven of nine episodes the MAP shortened (25 (4)\% 60 minutes before AF), developed a triangular shape, and the plateau amplitude decreased from 5.3 (1.2) to $2(0.2) \mathrm{mV}$. In the two remaining episodes the beat to beat variability of cycle length and MAP duration at $\mathbf{9 0 \%}$ repolarisation $\left(\mathbf{M A P d}_{90}\right)$ increased significantly from 24 (7) ms and 12 (8) ms (24 hours before AF) to 137 (27) $\mathrm{ms}$ and 56 (11) $\mathrm{ms}$ (30 minutes before AF) respectively. AF was successfully treated by the administration of sotalol in three cases and by a combination of verapamil and digoxin in a further four. Previously observed changes of $\mathrm{MAPd}_{90}$ and MAP morphology regressed after conversion to sinus rhythm.

Conclusions-The continuous and intermediate term recording of the MAP from atrial epicardium appears to be a valid tool for detecting imminent AF after cardiac surgery with a high sensitivity (99\%) and specificity (88\%). Optimised antiarrhythmic treatment may thus be given selectively for prophylaxis.

(Heart 1998;80:467-472)

Keywords: postoperative atrial fibrillation; monophasic action potential; postoperative management; prophylactic antiarrhythmic treatment; arrhythmias

Patients suffering from cardiac arrhythmias consume a significant proportion of medical resources. ${ }^{1}$ Of all the supraventricular tachyarrhythmias, atrial fibrillation is by far the most common and the most important. It affects up of $17 \%$ of individuals above the age of 70 years $^{2}$ and there is growing interest in the underlying mechanisms and the investigation of prophylactic treatment. Rhythm disturbances, mainly atrial fibrillation and flutter, also represent the major cause of patient morbidity and hospital cost following routine cardiac operations. ${ }^{1}$ The incidence is about $30 \%$ after coronary bypass surgery and up to $48 \%$ after aortic valve replacements. ${ }^{3}$ The pathophysiology of postoperative episodes of atrial fibrillation is still a matter of discussion, in contrast to ordinary atrial fibrillation, which has been attributed to various re-entry mechanisms. ${ }^{45}$ One aim of ongoing research is thus to elucidate the mechanism of postoperative arrhythmias. On the other hand in the clinical setting emphasis is placed upon finding a reliable method of identifying those patients who will go on to develop arrhythmias, so that specific prophylactic treatment can be started.

To understand the mechanisms and predict the onset of cardiac arrhythmias, we need a more detailed assessment of the electrical activity of the myocytes than can be obtained from the surface ECG. Signal averaged P wave analysis has been used to predict the onset of atrial fibrillation after bypass surgery and has been shown to be fairly specific $(80 \%)$ and sensitive $(75 \%)$ in identifying the patients at risk. ${ }^{6}$ Ideally, however, the cell action potential (CAP) should be followed to monitor physiological and pathological processes as well as the effects of pharmacological interventions at cell level. ${ }^{8}{ }^{9}$ Recording the CAP is currently a time limited, invasive procedure resulting in cell damage. ${ }^{10}$ Therefore, long term use in the clinical setting requires an indirect method that does not damage the cells. In this context the recording and evaluation of the monophasic action potential (MAP) from endocardial or epicardial surfaces may be useful. ${ }^{11}$ The MAP is the summed signal of the CAP values of a group of electrically active cells in the vicinity of the different electrode and thus closely resembles the CAP in its time course. $^{10}$ All kinds of global changes in depolarisation and repolarisation behaviourfor example, the development of a proarrhythmic substrate-thus become detectable and may in theory be monitored.

Up to now, the limitations of MAP recording techniques using the traditional $\mathrm{Ag} / \mathrm{AgCl}$ electrodes have been the lack of long term stability of the signal (less than three hours). ${ }^{911}$ With the advent of modern electrode coating techniques it has recently become possible to develop a biocompatible electrode material with biophysical properties identical to $\mathrm{Ag} /$ $\mathrm{AgCl}$ electrodes but with long term stability. ${ }^{12}{ }^{13}$ Fractally coated electrodes with 
Table 1 Baseline parameters of the patients included in the study

\begin{tabular}{|c|c|c|c|c|c|c|c|}
\hline Patient & $\begin{array}{l}\text { Age at } \\
\text { operation }\end{array}$ & Sex & Disease & $\begin{array}{l}\text { Date of } \\
\text { operation }\end{array}$ & Prosthesis & $\begin{array}{l}\text { Cross clamp } \\
\text { time (mins) }\end{array}$ & $\begin{array}{l}\text { MAP recording } \\
\text { (AF onset) (days) }\end{array}$ \\
\hline \multicolumn{8}{|c|}{ Patients who did not develop atrial fibrillation or flutter } \\
\hline 1 & 85 & $\mathrm{~F}$ & Aortic incompetence & 07.10 .94 & SJM 23 & 47 & 6 \\
\hline 2 & 61 & M & Aortic stenosis & 24.11 .94 & SJM 25 & 65 & 7 \\
\hline 3 & 64 & $\mathrm{~F}$ & $\begin{array}{l}\text { Aortic stenosis/aortic } \\
\text { incompetence }\end{array}$ & 12.12 .94 & SJM 23 & 70 & 6 \\
\hline 4 & 41 & M & $\begin{array}{l}\text { Aortic incompetence/ } \\
\text { aortic stenosis }\end{array}$ & 11.01 .95 & SJM 23 & 56 & 7 \\
\hline 5 & 29 & M & $\begin{array}{l}\text { Aortic incompetence/ } \\
\text { aortic stenosis }\end{array}$ & 01.03 .95 & (Ross) & 115 & 4 \\
\hline 6 & 72 & $\mathrm{~F}$ & Aortic stenosis & 04.04 .95 & CE 23 & 93 & 7 \\
\hline 7 & 85 & $\mathrm{~F}$ & Aortic stenosis & 19.05 .95 & CE 21 & 46 & 10 \\
\hline 8 & 58 & M & Aortic stenosis & 06.09 .95 & SJM 27 & 41 & 9 \\
\hline 9 & 56 & M & $\begin{array}{l}\text { Aortic incompetence/ } \\
\text { aortic stenosis }\end{array}$ & 10.10 .95 & SJM 27 & 58 & 8 \\
\hline 10 & 70 & M & Aortic incompetence & 20.10 .95 & SJM 23 & 102 & 7 \\
\hline 11 & 57 & M & Aortic incompetence & 11.03 .96 & SJM 27 & 52 & 7 \\
\hline 12 & 77 & M & Aortic incompetence & 29.04 .96 & SJM 27 & 39 & 8 \\
\hline 13 & 62 & M & Aortic stenosis & 21.05 .96 & SJM 23 & 59 & 9 \\
\hline 14 & 48 & M & Aortic incompetence & 16.09 .96 & SJM 25 & 53 & 7 \\
\hline 15 & 82 & $\mathrm{~F}$ & Aortic stenosis & 19.12 .96 & SJM 23 & 110 & 14 \\
\hline 16 & 72 & M & $\begin{array}{l}\text { Aortic incompetence/ } \\
\text { aortic stenosis }\end{array}$ & 09.11 .94 & Med 27 & 44 & 7 \\
\hline Mean & 63 & & & & & 66 & 8 \\
\hline \multicolumn{8}{|c|}{ Patients who did develop atrial fibrillation or flutter } \\
\hline 1 & 49 & $\mathrm{~F}$ & $\begin{array}{l}\text { Aortic stenosis/aortic } \\
\text { incompetence }\end{array}$ & 18.05 .95 & SJM 21 & 60 & $8(1)^{\star}$ \\
\hline 2 & 58 & $\mathrm{~F}$ & Aortic stenosis & 10.01 .96 & SJM 25 & 41 & $10(5,8)$ \\
\hline 3 & 50 & $\mathrm{~F}$ & Aortic incompetence & 13.03 .96 & SJM 27 & 78 & $9(2,3,5)$ \\
\hline 4 & 76 & M & $\begin{array}{l}\text { Aortic incompetence/ } \\
\text { aortic stenosis }\end{array}$ & 16.04 .96 & SJM 25 & 87 & $7(3)$ \\
\hline 5 & 73 & M & $\begin{array}{l}\text { Aortic stenosis/aortic } \\
\text { incompetence }\end{array}$ & 30.04 .96 & CE 23 & 40 & $6(2)$ \\
\hline 6 & 75 & $M$ & Aortic incompetence & 30.04 .96 & SJM 29 & 48 & $7(4)$ \\
\hline Mean & 65 & & & & & 64 & $8(3.5)$ \\
\hline
\end{tabular}

^Numbers in parentheses are days after surgery when atrial fibrillation or flutter occurred.

SJM, St Jude Medical; CE, Carpentier-Edwards; Med, Medtronic.

low impedance, no frequency dependent damping, and low polarisability allow reliable measurement of the MAP over a period of at least 14 days. $^{12}$ A comparison of the signal obtained with an $\mathrm{Ag} / \mathrm{AgCl}$ electrode and the fractally coated lead at the same location endocardially has shown a near perfect correlation $(r=0.99) .{ }^{14}$ Thus the observation of electrodynamic changes on the near-myocyte level in vivo has become feasible.

The underlying hypothesis of the work presented here is that the electrical alterations that culminate in the occurrence of postoperative atrial arrhythmias (development of the substrate) are reflected by gradual changes in the cellular action potentials and therefore in the atrial MAP (aMAP). Specific changes in the time course of the aMAP recorded from human epicardium should thus be a valid indicator to predict the onset of atrial fibrillation and consequently allow patient specific prophylactic treatment to be started.

\section{Methods}

After giving written consent in accordance to the declaration of Helsinki, 22 patients (eight female, 14 male, mean (SD) age 64 (12) years, range 29 to 85 ) undergoing aortic valve replacements were included in this retrospective study. At the time of the operation none of the patients was receiving antiarrhythmic drugs, nor did they have any relevant accompanying diseases such as diabetes mellitus or renal failure. Baseline variables are shown in table 1 . The intraoperative presence of electrically non-convertible atrial fibrillation or flutter at the end of the period of extracorporeal circulation was a criterion for exclusion. Patients receiving coronary artery bypass grafts or mitral valve replacements were also excluded (see Discussion).

After the aortic valve replacement, bipolar epicardial MAP electrodes ${ }^{13}{ }^{15}$ (Biotronik, Berlin, Germany) were attached to the lateral aspect of the right atrium and the apex of the right ventricle using three $6 / 0$ prolene sutures in each case. The leads were exteriorised transcutaneously like conventional temporary pacing wires. From the time the patients arrived on the intensive care unit the MAPs were recorded using an isolation amplifier with adjustable amplification (range \pm 5 to $\pm 250 \mathrm{mV}$ ), digitised $(500 \mathrm{~Hz}$ sampling frequency, 12 bit resolution), and stored on hard disk. For the duration of the inpatient stay the signals were recorded almost continuously. The MAP leads were removed by traction before the patient was discharged from hospital.

At the onset of atrial fibrillation or flutter (AF) the patients received one of the institution's standard antiarrhythmic drug regimens (verapamil/digoxin intravenously or sotalol orally).

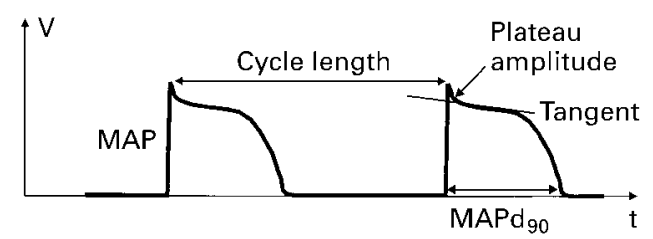

Figure 1 Atrial monophasic action potential (MAP) parameters analysed using the semiautomatic evaluation program. $M A P d_{90}=M A P$ at $90 \%$ repolarisation. 
In the verapamil/digoxin group, patients initially received an intravenous bolus dose of 5 to $15 \mathrm{mg}$ of verapamil and the first of three intravenous doses of $0.4 \mathrm{mg}$ digoxin (at eight hour intervals) to control the heart rate. This was followed by a continuous infusion of verapamil for 24 to 48 hours, adjusted according to heart rate, after which oral administration was begun at a dose of $80 \mathrm{mg}$ three times daily. Digoxin was given according to plasma digoxin levels.

Patients in the sotalol group received the drug orally after an initial intravenous dose if the heart rate was above 140 beats/min. The dose was then increased until heart rate fell sufficiently or until the mean blood pressure became low enough to cause symptoms. Sotalol was continued on a thrice daily regimen up to a dose of 120 to $240 \mathrm{mg}$ /day.

For both groups the antiarrhythmic treatment was continued prophylactically after cardioversion for several weeks.

The standard MAP parameters (cycle length, MAP duration at $20 \%, 50 \%$, and $90 \%$ repolarisation $\left(\mathrm{MAPd}_{25 / 50 / 90}\right)$, and plateau amplitude $)^{9}{ }^{10}$ were evaluated retrospectively using semiautomatic MAP evaluation software (fig 1). The plateau amplitude was taken as the intercept of the initial upstroke and a tangent placed on the plateau. From these parameters the MAP duration normalised for cycle length $\left(\mathrm{nMAPd}_{90}=\mathrm{MAPd}_{90} /\right.$ cycle length $)$ and the difference between $\mathrm{nMAPd}_{90}$ and $\mathrm{nMAPd}_{25}$ were calculated. Furthermore the beat to beat variability of cycle length $(\triangle \mathrm{CL})$ and MAP duration $(\triangle \mathrm{MAPd})$ were determined. The simple normalisation procedure which we used for MAP duration, to allow for a comparison at different heart rates and between patients, is based on the well documented finding that in the range of 50 to 120 beats/min the correlation between $\mathrm{MAPd}_{90}$ and heart rate is linear. ${ }^{10}{ }^{16}$

The occurrence of atrial fibrillation or flutter was correlated with the nMAPd, the $n \mathrm{MAPd}_{90-25}$, the plateau amplitude, and the variability parameters. For each patient the MAP parameters outlined above were prima-
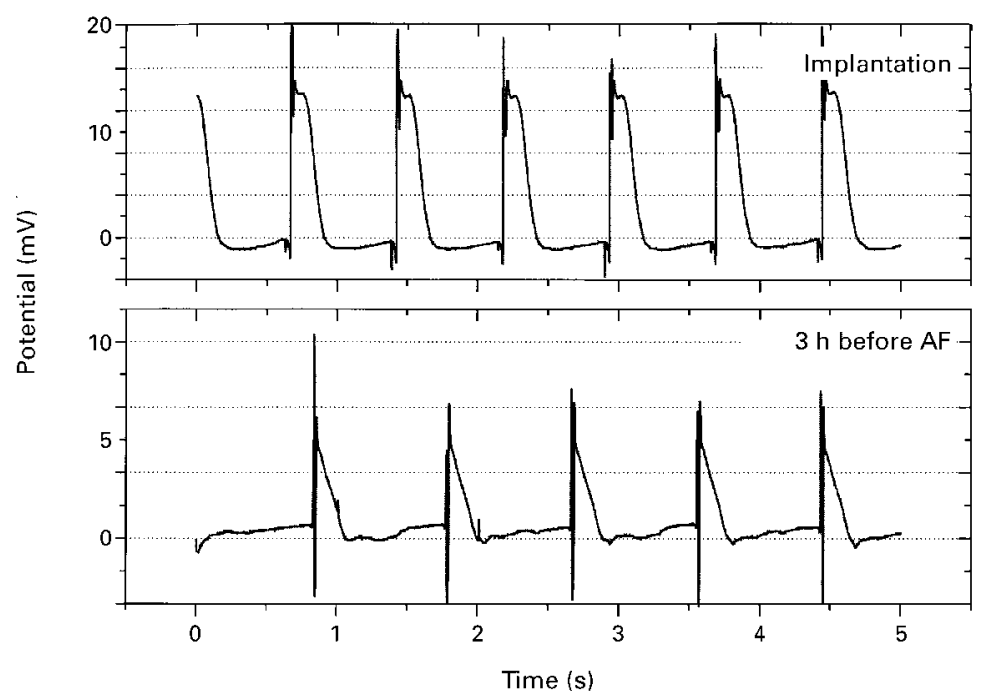

Figure 2 Atrial monophasic action potential before the onset of atrial fibrillation (AF). rily plotted against time. If $\mathrm{AF}$ occurred, the MAP changes were analysed retrogradely with reference to the time of onset and compared between patients. Measurements at 24 hours, three hours, and 30 minutes before $\mathrm{AF}$ (chosen arbitrarily) were averaged over three minutes and compared with those in patients who did not develop $\mathrm{AF}$ in that overall time window.

In those patients in whom sinus rhythm was successfully re-established by pharmacological treatment, the aMAPs were compared with those recorded before the onset of AF.

Generally, measurements were only accepted if there was a constant baseline, indicating a stable electrode position.

\section{Results}

In all 22 patients the MAPs were recorded with a signal to noise ratio of $>26 \mathrm{~dB}$ (fig 2). Plateau amplitudes started at $5.3(1.2) \mathrm{mV}$ in the atrium $(\mathrm{n}=21)$ and $15(7) \mathrm{mV}$ in the ventricle $(n=5)$. After an average time of 48 hours the amplitudes stabilised at $40-60 \%$ of the initial values. This development correlated with the cessation of fluid loss over the pericardial drains. In an earlier published control study, this change in amplitude was shown not to affect the time course of the MAPs.

The longest recording period was 14 days in a patient who remained in hospital for pulmonary complications. At that time the atrial signal measured $2.3 \mathrm{mV}$. All other patients left the hospital between five and 10 days postoperatively.

During the cumulative recording period of 170 days (mean 8.0 (2.7) days, range 4 to 14 ) no episodes of sustained ventricular arrhythmias but nine episodes of AF were observed. In seven cases these occurred within the first 72 hours after surgery.

Several hours before the occurrence of atrial fibrillation or atrial flutter the morphology of the aMAP was found to have changed significantly compared with the initial recordings. In seven of nine cases the aMAP developed a typically triangular shape (fig 2).

A heart rate independent shortening of $\mathrm{MAPd}_{90}$ and $\mathrm{MAPd}_{50}$ was observed $\left(\mathrm{nMAPd}_{90}\right.$ : -25 (4)\%; nMAPd $_{50}:-22 \%$ ) 60-180 min before AF. The difference $n M A P d_{90}-n_{M A P d}$, which

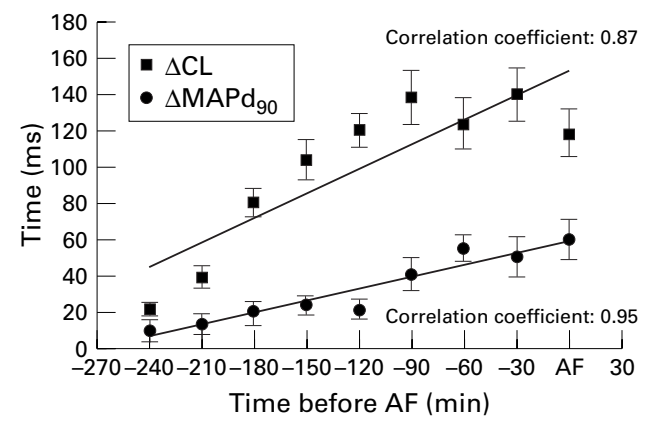

Figure 3 Changes in cycle length and monophasic action potential $(M A P)$ duration variability and their linear correlation with the time interval before the onset of atrial fibrillation or flutter $(A F)$. Error bars $=S D$. $\triangle C L$, cycle length variability, $\triangle M A P d_{90}$, variability of monophasic action potential duration at $90 \%$ repolarisation. 


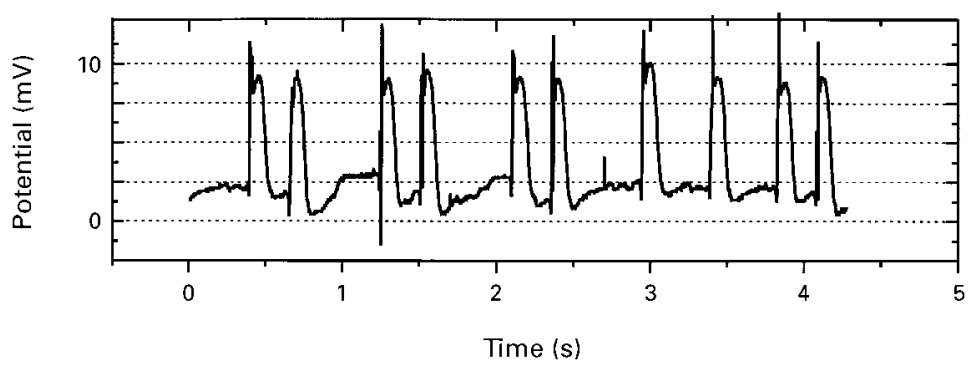

Figure 4 Atrial monophasic action potential morphology before atrial fibrillation.

Table 2 Average changes of normalised monophasic action potential duration ( $\mathrm{MAP})$, triangularity $\left(M A P d_{00-20}\right)$, variability of cycle length $(\triangle C L)$, and variability of $M A P$ duration ( $\left.\triangle M A P d_{90}\right)$ before onset of atrial fibrillation $(A F)$ compared with changes in patients without $A F$

\begin{tabular}{|c|c|c|}
\hline Time before $A F$ & No $A F$ & $A F$ \\
\hline \multicolumn{3}{|l|}{ nMAPd $_{90}$} \\
\hline-24 hours & $421(26) \mathrm{ms}$ & $398(36) \mathrm{ms}$ \\
\hline-3 hours & & $354(45) \mathrm{ms}$ \\
\hline-30 minutes & $p \leqslant 0.01$ & 296 (31) ms \\
\hline \multicolumn{3}{|l|}{$\Delta \operatorname{MAPd}_{90}$} \\
\hline-24 hours & $13(6) \mathrm{ms}$ & $12(8) \mathrm{ms}$ \\
\hline-3 hours & & $23(13) \mathrm{ms}$ \\
\hline-30 minutes & $p \leqslant 0.05$ & 56 (11) ms \\
\hline \multicolumn{3}{|l|}{ MAPd $_{90-25}$} \\
\hline-24 hours & $37(6) \mathrm{ms}$ & 40 (9) ms \\
\hline-3 hours & & $59(10) \mathrm{ms}$ \\
\hline-30 minutes & $p \leqslant 0.001$ & 99 (16) ms \\
\hline \multicolumn{3}{|l|}{$\Delta \mathrm{CL}$} \\
\hline-24 hours & $20(6) \mathrm{ms}$ & $24(6) \mathrm{ms}$ \\
\hline-3 hours & & $122(10) \mathrm{ms}$ \\
\hline-30 minutes & $p \leqslant 0.001$ & $137(17) \mathrm{ms}$ \\
\hline
\end{tabular}

Values are mean (SD).

reflects the triangularity of the aMAP, increased by $63(9) \%$. The plateau amplitude of the atrial MAP decreased from $5.3(1.2)$ to $2(0.2) \mathrm{mV}$ (fig 2).

In the other two cases the beat to beat changes in cycle length $(\triangle \mathrm{CL})$ and beat to beat aMAP duration $(\triangle \mathrm{MAPd})$ increased significantly (linear correlation coefficient; fig 3 ) with concurrent appearance of a bigeminal picture in one case (fig 4).

Table 2 summarises the changes described in comparison with those patients who did not develop AF. Statistical significance (Wilcoxon rank-sum (Mann-Whitney) test for two independent samples) was reached in all cases, the

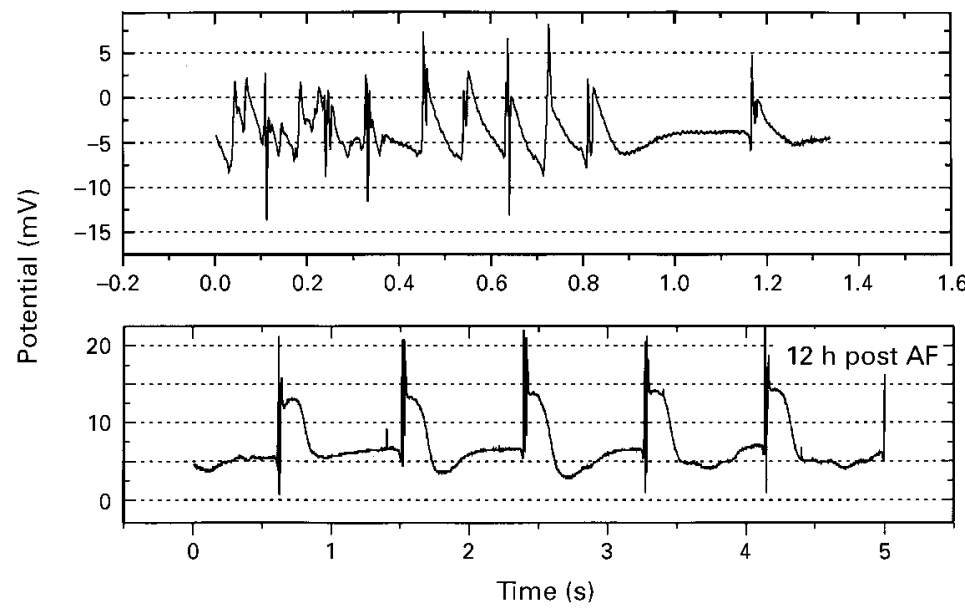

Figure 5 Atrial monophasic action potential morphology after the conversion of atrial fibrillation to sinus rhythm. null hypothesis being rejected with the $\mathrm{p}$ values shown in table 2 .

The majority of patients (73\%) did not experience sustained atrial or ventricular arrhythmias during the observation period. Beat to beat changes of the cycle length and the $\mathrm{MAPd}_{90}$ did not exceed $\triangle \mathrm{CL}=30 \mathrm{~ms}$ and $\triangle \mathrm{MAPd}=20 \mathrm{~ms}$ as compared with those cases where AF developed (table 2). Alterations of the morphology to a triangular shape were observed in two of these patients without the progression to sustained AF.

$\mathrm{AF}$ was successfully treated with sotalol in three cases and with combination of verapamil and digoxin in four. Two of the patients were still in $\mathrm{AF}$ at the end of the observation period.

After the conversion to sinus rhythm a triangular shaped aMAP was seen in all cases, with a duration of 305 (40) ms (fig 5, upper trace). The aMAP plateau phase returned 4-12 hours thereafter (fig 5, lower trace). The previously observed reduction of $\mathrm{nMAPd}_{90}$ was seen to regress (30 minutes after AF: -20 (12)\%; 60 minutes after AF: -19 (5)\%; three hours after AF: $-11(3) \%)$. The rate corrected aMAP duration of those patients where AF was treated successfully with sotalol showed a heart rate independent prolongation beyond prearrhythmic values $\left(\mathrm{nMAPd}_{90}: 11 \%\right)$.

\section{Discussion}

Using new fractally coated temporary pacing leads, prolonged recordings of the epicardial MAP are possible for up to 14 days with an acceptable signal to noise ratio, as reported elsewhere. ${ }^{13} 14$ The morphology and standard MAP parameters correlate very well with those obtained with $\mathrm{Ag} / \mathrm{AgCl}$ electrodes (the gold standard) in the acute setting. ${ }^{14} \mathrm{~A}$ decrease in signal amplitude was observed in all cases during the first 48 hours. This is assumed to be caused by alterations in the composition of the pericardial fluid around the different electrode, as well as to changes in pressure on the electrode. The latter depend on the amount of pericardial fluid and the ventilator settings during the period of artificial ventilation and are well known to change MAP signal size. ${ }^{10}$ Samples of the fluid collected from the pericardial drains deviate from extracellular fluid in terms of ionic concentrations by up to one order of magnitude and may thus be responsible for changes in the size of transmembrane currents and therefore the MAP at the epicardial surface. One result of the initial fall in signal size with time is that the marked decline in plateau amplitude observed before the onset of atrial fibrillation becomes a less specific predictor of atrial fibrillation and we have thus omitted it from the analysis in this first approach. In patients who did not develop atrial fibrillation the morphology of the MAP recorded with the fractally coated leads remained stable despite the decrease in amplitude. ${ }^{13}{ }^{15}$ Thus an evaluation of the time course of the signals remains perfectly valid. In conclusion we feel that there is sufficient evidence to justify our approach of using fractally coated leads to monitor the MAP 
continuously and thereby gain insight into the pathophysiological mechanisms underlying the onset of atrial fibrillation after cardiac surgery.

Only patients operated on for aortic valve pathology were included in this first study. There were two principal reasons for excluding patients undergoing bypass surgery. First, it was felt that the removal of the MAP electrode might sever a venous graft running anterior to the right coronary artery. Second, the presence of ischaemic disease of the myocardium, especially in the area of supply of the right coronary artery, introduces additional variability with respect to the interpretation of the pathophysiological mechanisms. In our current prospective and randomised approach, patients with coronary artery bypass grafts are also included.

The results point to two apparently different mechanisms for developing the proarrhythmic substrate. In seven of our nine cases of postoperative atrial fibrillation the aMAPs shortened, with concomitant loss of the plateau phase within hours of the onset of atrial fibrillation. Among the remaining 16 patients only two showed similar changes in their atrial MAPs without presenting with atrial fibrillationor flutter. In these cases the changes in aMAP were less pronounced but were associated with an increased incidence of atrial extrasystoles, couplets, and triplets. It thus appears that the morphological changes in the aMAP observed here are a good indicator that the atrial rhythm is likely to become unstable and to deteriorate to atrial fibrillation. By analogy, similar alterations of the MAP morphology have been described in experimental settings where hypoxic/ischaemic, hypokalaemic (intracellular), and acidotic changes in the extracellular fluid were observed in connection with the onset of ventricular arrhythmias. $^{81718}$ From clinical experience these factors are also well known to encourage the proarrhythmic substrate for atrial fibrillation in the postoperative setting.

Shortening of the MAP with an accompanying reduction in the refractory period ${ }^{19}$ appears to represent a precursor of the remodelling process described by Allessie. In terms of the underlying mechanism, in the postoperative setting there appears to be a combination of autonomic imbalance and intracellular ionic dysregulation (which might be cardioplegia dependent).

It has been reported that the MAP alternans observed in the remaining two patients who progressed to atrial fibrillation is associated with an increased likelihood of dysrhythmias. ${ }^{20}$ In the present context this represents another piece of evidence for the increased instability of the atrial myocardium before atrial fibrillation occurs. It appears that the phenomenon is caused either by a regional disturbance remote from our recording site or by a global change in intra-atrial or interatrial conduction that is largely independent of the cellular action potential.

All episodes of atrial fibrillation observed here were preceded either by morphological alterations of the aMAP or an increased beat to beat variability of cycle length and MAP duration. Thus the rate corrected MAP duration,
Table 3 Parameters and the corresponding limits for the prediction of atrial fibrillation after aortic valve replacement

\begin{tabular}{ll}
\hline Parameter & Prediction threshold \\
\hline nMAPd $_{90}$ & Reduction by $20 \%$ \\
MAPd $_{90-25}(\mathrm{~ms})$ & Increase by $20 \%$ \\
$\Delta \mathrm{CL}$ & Increase over $75 \mathrm{~ms}$ \\
$\Delta \mathrm{MAPd}_{90}$ & Increase over $20 \mathrm{~ms}$
\end{tabular}

CL, cycle length; MAPd $_{90}$, monophasic action potential duration at $90 \%$ repolarisation; $\mathrm{MAPd}_{90-25}$, triangularity of monophasic action potential; $n$, normalised.

the triangularity measure $\mathrm{MAPd}_{90-25}$, and the variability parameters may all be used to predict atrial fibrillation after aortic valve replacements. The preliminary prediction threshold for each parameter may be chosen as shown in table 3 . Using these limits for the parameters, the sensitivity of the method for predicting atrial arrhythmias would be $99 \%$ with a specificity of $88 \%$.

After pharmacological cardioversion, restitution of the typical shape of the aMAP was observed in all the four patients in whom it had been triangular. As the drug regimens, individual doses, and the duration of atrial fibrillation were very different in these cases, reference is made only to experimental work where similar observations have been reported in patients converted from atrial fibrillation to sinus rhythm under MAP monitoring. ${ }^{16}{ }^{19}$ The possibility of using the MAP for drug treatment monitoring in this context is well recognised ${ }^{10}$ and presents part of our ongoing study.

Other methods of monitoring the emergence of a substrate for atrial fibrillation or flutter after cardiac surgery have focused on the signal averaged $P$ wave duration. ${ }^{67}$ The results obtained in these studies also highlight the value of observing changes in atrial electrophysiology for predicting the onset of atrial fibrillation. Whereas we report a shortening of the repolarisation phase of the atrial myocyte action potential, signal averaged $\mathrm{P}$ wave duration has been shown to increase before the onset of atrial fibrillation. We feel that these results are not necessarily contradictory. Even if the action potential and the refractory period of the myocytes is reduced, conduction throughout the atria may well be prolonged. In fact it could be argued that the combination of the two effects would result in far greater susceptibility to the development of re-entry circuits. Our future approach includes an analysis of the intra-atrial conduction velocity in order to address this question.

\section{LIMITATIONS}

We recognise several limitations of this clinical and primarily phenomenological approach to postoperative atrial fibrillation. The data were evaluated retrospectively. To be of use in the clinical setting for prophylaxis and for monitoring the therapeutic result an on-line semiautomatic evaluation system is required. This forms the basis of a prospective study presently underway with the prediction thresholds outlined above.

A further point of criticism is the small number of observed episodes of atrial fibrillation, which imposes a limit on the statistical 
significance of the changes observed. Lastly we recognise the focal nature of the data acquisition from the myocardium, so that a multifocal approach is presently being used.

CONCLUSIONS

We conclude that intermediate term monitoring of the atrial MAP represents a useful tool for the management of patients after cardiac surgery, which should aid in the reduction of morbidity and hospital costs. The results also suggest that there is a place for this technology in on-line evaluation of pharmacological treatment on a long term basis.

1 Aranki SF, Shaw DP, Adams DH, et al. Predictors of atrial fibrillation after coronary artery surgery. Current trends
and impact on hospital resources. Circulation 1996;94:3907 .

2 Campbell RWF. Supraventricular tachycardia. Eur Heart $\mathcal{f}$ 1996;17:C:21-5.

3 Cresswell LL, Schuessler RB, Rosenbloom M, et al. Hazards of postoperative atrial arrhythmias. Ann Thorac Surg 1993; 56:539-49

4 Allessie MA, Kirchhof CJHJ, Konings KTS. Unravelling the electrical mysteries of atrial fibrillation. Eur Heart $\mathcal{F}$ 1996;17:2-9.

5 Konongs KTS, Kirchhoff CJHJ, Wellens HJ, et al. High-density mapping of electrically induced atrial fibrillation in humans. Circulation 1994;89:1665-80.

6 Stafford PJ, Kolvekar S, Cooper J, et al. Signal averaged P wave compared with standard electrocardiography or echocardiography for prediction of atrial fibrillation after coronary bypass grafting. Heart 1997;77:417-22.

7 Zaman AG, Alamgir F, Richens T, et al. The role of signal averaged $\mathrm{P}$ wave duration and serum magnesium as a comaveraged $\mathrm{P}$ wave duration and serum magnesium as a com-
bined predictor of atrial fibrillation after elective coronary artery surgery. Heart 1997;77:527-31.
8 Ten Eick RE, Whalley DW, Rasmussen HH. Connections: heart disease, cellular electrophysiology, and ion channels. FASEB f 1992;6:2568-80.

9 Yuan S, Blomström-Lundqvist C, Olsson SP. Monophasic action potentials: concepts to practical applications. $7 \mathrm{Car}$ diovasc Electrophysiol 1984;5:287-308.

10 Franz MR. Method and theory of MAP recording. Prog Cardiovasc Dis 1991;6:347-68.

11 Franz MR. Bridging the gap between basic and clinically electrophysiology: what can be learned from monophasic action potential recordings? I Cardiovasc Electrophysiol 1994;5:699-710.

12 Bolz A, Hubmann M, Hardt R, et al. Low polarization pacing lead for detecting the ventricular evoked response. Med Prog Technol 1993;19:129-37.

13 Pichlmaier AM, Lang V, Harringer W, et al. Long-term recordings of the monophasic action potential using new implantable pacing leads [abstract]. PACE 1995;18:1094.

14 Hubmann $M$, Wetzig $T$, Fröhlich $R$, et al. MAPmeasurement - a comparison of $\mathrm{Ag} / \mathrm{AgCl}$ and fractally coated pacing leads [abstract]. Eur $\mathcal{F}$ Clin Physiol Electrophysiol 1996;6:213.

15 Lang V, Pichlmaier AM, Harringer W, et al. Long term recordings of the monophasic action potential from human epicardium [abstract]. Eur 7 Clin Physiol Electrophysiol 1996,6:110

16 Franz MR, Li C, Karasik P, et al. Abnormal action potential duration/cycle length relation in patients cardioverted from chronic atrial fibrillation [abstract]. Circulation 1995;92: 404.

17 John RM, Taggart PI, Sutton PM, et al. Endocardial monophasic action potential recordings for the detection of myocardial ischaemia in man: a study using atrial pacing stress and myocardial perfusion scintigraphy. Am Heart $\mathscr{f}$ 1991;122:1599-609.

18 Janse MJ, Kléber AG. Electrophysiological changes and ventricular arrhythmias in the early phase of regional myocardial ischaemia. Circ Res 1981;49:1069-81

19 Daoud EG, Niebauer M, Bogun F, et al. Atrial fibrillation induced shortening of atrial refractoriness in humans [abstract]. Circulation 1995;92:404.

20 Antzelevitch C, Sicouri S, Litovsky SH, et al. Heterogeneity within the ventricular wall. Circ Res 1991;69:1427-49. 